\title{
Distúrbios neurológicos em trabalhadores com baixos níveis de chumbo no sangue. II-Disfunções neurocomportamentais*
}

\section{Neurological disturbances in workers with low levels of lead in the blood. II-Neuropsychological disorders}

\author{
Ricardo Cordeiro, Euclydes C. Lima Filho, Paulo E. T. Salgado, Cleide Oliveira Santos, Léa \\ Constantino e Maria Luiza Leão Salerno Malatesta
}

Departamento de Saúde Pública da Faculdade de Medicina de Botucatu. Botucatu, SP - Brasil (R.C.); Departamento de Epidemiologia e Métodos Quantitativos da Escola Nacional de Saúde Pública. Rio de Janeiro, RJ - Brasil (E.C.L.F.); Departamento de Princípios Ativos Naturais e Toxicologia da Faculdade de Ciências Farmacêuticas de Araraquara. Araraquara, SP - Brasil (P.E.T.S.); Centro de Referência para a Saúde do Trabalhador de São Bernardo do Campo. São Bernardo do Campo, SP Brasil (C.O.S., L. C., M.L.L.S.M.)

\begin{abstract}
Resumo
Trata-se de um estudo observacional, transversal, com população de estudo aleatorizada, cujo objetivo foi contribuir para a avaliação da validade dos limites de tolerância biológica (LTB) estabelecidos no Brasil para a plumbemia $(\mathrm{Pb}-\mathrm{S})$ e a excreção urinária do ácido $\delta$-aminolevulínico (ALA-U). Para tanto, foi aplicada uma variação do WHO Neurobehavioral Core Test Battery a um grupo de trabalhadores expostos ao chumbo, cujos valores de $\mathrm{Pb}-\mathrm{S}$ e ALA-U encontravam-se no momento - bem como nos 2 anos precedentes - abaixo dos LTB brasileiros. Os resultados foram comparados aos obtidos por intermédio da aplicação da mesma bateria de testes, em um grupo-controle não exposto ao chumbo. No grupo exposto foram encontrados sinais de comprometimento da memória, humor e coordenação motora fina - que diferiam significativamente dos resultados obtidos no grupo-controle $(\mathrm{p}=0,02)$. Os resultados apontam contra a validação dos LTB estabelecidos no Brasil para a Pb-S e a ALA-U.
\end{abstract}

Manifestações neurológicas. Intoxicação por chumbo, prevenção \& controle. Exposição ocupacional, normas. Atividade nervosa superior.

\begin{abstract}
This is a cross-sectional study with a randomized choice of individuals aiming at studying the validity of the Brazilian biological exposure limits applied to lead level in the blood $(\mathrm{PbB})$ and delta-aminolevulinic acid in the urine (ALAU), which are $60 \mu / d l$ and $10 \mathrm{mg} / g$.creat., respectively. Thus, twenty
\end{abstract}

\footnotetext{
* Financiado pela Fundação para o Desenvolvimento da UNESP. (Processo FUNDUNESP 277/94-DFP/F/CBS e pelo Conselho Nacional de Desenvolvimento Científico e Tecnológico/CNPq. Processo 141069/92-9.

Correspondência para/Correspondence to: Ricardo Cordeiro - Caixa Postal 543 - 18618-970 Botucatu SP - Brasil - Fax: (014) 822.0421. E-mail: cordeiro@fmb.unesp.br

Recebido em 10.8.1995. Reapresentado em 21.2.1996. Aprovado em 13.3.1996.
} 
workers, whose $\mathrm{PbB}$ and ALAU values have been below these limits over the past two years, were selected at random at a battery plant in the State of $S$. Paulo, Brazil. The workers were submitted to a variation of the WHO Neurobehavioral Core Test Battery. The results were compared with those obtained for workers of a control group also chosen at random. The lead workers showed memory, mood and motor coordination disorders. Comparing these results with those obtained from the control group, a significant difference was observed ( $p$-value $<0.02$ ). The results indicate that the Brazilian biological exposure limits above should be reconsidered.

Neurologic manifestations. Lead poisoning, prevention and control. Occupational exposure, standards. Higher nervous activity.

\section{INTRODUÇÃO}

Muito embora não se disponha de informações sistematizadas a respeito da prevalência da intoxicação profissional pelo chumbo (IPPb) - bem como de todas as doenças ocupacionais - em nosso meio, supõe-se, a partir de relatos isolados e do conhecimento do parque industrial brasileiro, que ela seja relativamente alta $^{12}$.

Os limites de tolerância biológica (LTB) aplicados aos indicadores biológicos sensíveis à exposição ao chumbo assumem papel relevante na prevenção da IPPb.

Cordeiro e col. ${ }^{14}$ (1996) estudaram a validade dos atuais LTB aplicados aos dois indicadores biológicos de maior importância na prevenção da IPPb: concentração sangüínea de chumbo $(\mathrm{Pb}-\mathrm{S})$ e concentração urinária do ácido $\delta$-aminolevulínico (ALA-U). Os valores destes LTB foram estabelecidos pelo Ministério do Trabalho em 1983, por intermédio do Anexo II da Norma Regulamentadora $n^{\circ} 7^{9}$, como $60 \mu \mathrm{g} /$ dl e $15 \mathrm{mg} / \mathrm{l}$, respectivamente. Recentemente, o Ministério do Trabalho complementou essa Norma, por intermédio da Portaria $n^{\circ} 24 / 1994^{8}$, onde o LTB aplicado à ALA-U é alterado de $15 \mathrm{mg} / \mathrm{L}$ para $10 \mathrm{mg} /$ g.creat, mantendo inalterado o LTB aplicado à $\mathrm{Pb}-\mathrm{S}$. Nessa Portaria, o termo limite de tolerância biológica foi substituído por índice biológico máximo permitido (IBMP). O referido trabalho questiona a adequação dos valores acima citados.

É sabido que distúrbios neurocomportamentais se associam à exposição ocupacional ao chumbo em níveis inferiores àqueles responsáveis pelas alterações neurológicas periféricas ${ }^{6,7}$. Entretanto, a literatura especializada internacional refere apenas um estudo demonstrando a concomitância de disfunções nervosas centrais e periféricas em um mesmo grupo de trabalhadores expostos a baixos níveis de chumbo ${ }^{2,3}$.

No presente trabalho, aplicaram-se testes neurocomportamentais aos mesmos indivíduos estudados eletroneurograficamente por Cordeiro e col. ${ }^{14}$ (1996), objetivando-se também contribuir para o estudo da validade dos LTB estabelecidos no Brasil para a $\mathrm{Pb}$ $\mathrm{S}$ e a ALA-U.

\section{MATERIAL E MÉTODO}

\section{Hipótese de Trabalho}

Foi testada a seguinte hipótese:

Hipótese $H_{0}$ : Não ocorre alteração neurocomportamental em trabalhadores ocupacionalmente expostos ao chumbo inorgânico que apresentam $\mathrm{Pb}-\mathrm{S}$ e ALA-U abaixo dos LTB brasileiros.

A alteração neurocomportamental a que a hipótese $\mathrm{H}_{0}$ se refere foi definida como distúrbio no desempenho de no mínimo um dos testes ou subtestes abaixo, cuja natureza será discutida adiante:

- Profile of Mood State (POMS)

- Wechsler Memory Scale (WMS)

- Teste Memória Auditiva Imediata - Beatriz Lefèvre

- Subtestes Disco, Pontilhagem e Contas do teste Bateria Mecânica Léon Walther

- Teste Atenção Concentrada - Suzy Vijande Cambraia

- Subteste Digit Symbol (DSY) do Wechsler Adult Intelligence Scale (WAIS)

\section{Análise}

A hipótese acima foi testada por intermédio da comparação das médias dos resultados obtidos nas avaliações neurocomportamentais pelos trabalhadores expostos ao chumbo com as médias obtidas pelos trabalhadores do grupo não exposto. Tal comparação deu-se através da aplicação de testes t de Student, monocaudais, para diferenças entre médias de populações independentes, com nível de significância e poder do teste t previamente estabelecidos como 0,05 e 0,80 , respectivamente.

\section{Processo Amostral}

Foram amostrados dois grupos profissionais comparáveis, que diferiam quanto à exposição ao chumbo.

O grupo exposto foi alocado por intermédio de amostragem aleatória simples, tendo como base populacional 
a linha de montagem de uma fábrica de baterias de médio porte (280 trabalhadores) no interior do Estado de São Paulo. Após a exclusão dos trabalhadores com potenciais danos neurológicos não decorrentes da exposição ao chumbo, o grupo foi composto por 20 operários cujos indicadores $\mathrm{Pb}-\mathrm{S}$ e ALA-U encontravam-se, nos últimos dois anos, abaixo dos LTB estabelecidos no Brasil.

O grupo não exposto foi alocado por intermédio de amostragem aleatória estratificada, tendo como base populacional os soldadores de uma montadora de grande porte (2.400 trabalhadores) de veículos de transporte coletivo no interior do Estado de São Paulo. Também após a exclusão dos trabalhadores com potenciais danos neurológicos não decorrentes da exposição ao chumbo, o grupo foi composto por 20 operários que nunca se expuseram ocupacionalmente ao metal.

A magnitude da exposição e do efeito do chumbo nos dois grupos foi avaliada por intermédio da realização das seguintes análises toxicológicas: dosagem de $\mathrm{Pb}-\mathrm{S}$, ALA-U e atividade da enzima $\delta$-aminolevulínico desidratase (ALAD).

A equivalência dos dois grupos foi conferida por intermédio da comparação dos resultados obtidos pelos trabalhadores expostos e não expostos no seguinte conjunto de avaliações clínicas e laboratoriais:

- exame antropométrico e aferição da pressão arterial

- dosagens séricas de sódio, potássio, uréia, creatinina, TGO, TGP, y-GT, fosfatase alcalina, bilirrubina direta, bilirrubina indireta, triglicérides, colesterol, glicemia de jejum e urina tipo I.

O detalhamento dos procedimentos relativos ao processo amostral acima descrito, bem como das análises toxicológicas e dos exames gerais aplicados aos trabalhadores do dois grupos, encontram-se descritos em trabalho anterior (Cordeiro e col. ${ }^{14}$, 1996).

\section{Análises Neurocomportamentais}

Os grupos exposto e não exposto foram submetidos aos testes e subtestes referidos no item "hipótese de trabalho".

Não existe ainda, em nosso meio, um conjunto bem estabelecido de testes para diagnosticar disfunções nervosas superiores relacionadas à ação do chumbo. $\mathrm{O}$ conjunto anteriormente descrito é uma adaptação às populações trabalhadoras brasileiras do conjunto de testes elaborado por um comitê internacional de especialistas em neurotoxicologia, reunido em 1983 sob patrocínio da Organização Mundial de Saúde, formado com o intuito de construir um instrumento diagnóstico para disfunções neurocomportamentais em trabalhadores expostos a agentes neurotóxicos ${ }^{19}$. A adaptação brasileira foi feita por um grupo de profissionais que contou com a participação de psicólogos do Centro de Referência para Saúde do Trabalhador da Secretaria Municipal de Saúde de São Bernardo do Campo (SP).
O teste Profile of Mood States ${ }^{17}$ foi desenvolvido com o intuito de descrever e mensurar o estado de humor e a afetividade dos analisados. O teste é composto pelos subtestes Tensão, Depressão, Raiva, Vigor, Fadiga e Confusão. Consiste de um conjunto de 65 questões sobre diferentes aspectos do humor e afetividade, acompanhadas cada uma delas de uma escala de 5 pontos, onde solicita-se aos analisados que marquem, passo a passo, o resultado que melhor exprime seu estado na última semana.

O teste Wechsler Memory Scale avalia componentes da memória do analisado, checando sua capacidade de retenção e posterior lembrança de histórias curtas ${ }^{19}$.

$\mathrm{O}$ teste Memória Auditiva Imediata foi criado e padronizado por Lefèvre* para avaliar a integridade dos processos implicados na memorização auditiva imediata. Consiste do enunciado de séries crescentes de dígitos, onde solicita-se ao analisado que repita a série, ora na ordem enunciada, ora na ordem inversa.

Os subtestes Disco, Pontilhagem e Contas, do teste Bateria Mecânica Léon Walther ${ }^{4}$ consistem da cronometragem da realização completa de uma série de tarefas manuais padronizadas, avaliando assim a habilidade manual, a rapidez de movimentos e a coordenação motora do analisado.

O Teste Atenção Concentrada $\left(\right.$ Cambraia $\left.^{10}\right)$ foi criado e padronizado para a mensuração do grau de atenção e concentração. Consiste numa sequiência de tamanho 441 de 12 símbolos diferentes, solicitando-se ao analisado, num tempo pré-determinado, que marque todas as ocorrências (147 ao todo) de 3 dos 12 símbolos, escolhidos previamente ao acaso.

O subteste Digit Symbol do teste Wechsler Adult Intelligence Scale avalia a velocidade do processo percepção/motricidade. Nele solicita-se ao analisado que associe, em um tempo pré-determinado, uma série de dígitos a um conjunto de símbolos abstratos, de acordo com um gabarito fornecido ${ }^{19}$.

Os exames neurocomportamentais foram todos aplicados em salas padronizadas, nas empresas, durante o expediente de trabalho, individualmente conduzidos por profissionais psicólogos especializados e familiarizados com os testes.

\section{RESULTADOS}

Não foram encontradas diferenças significativas $(\alpha=0,05)$ ao camparar-se os grupos exposto e não exposto por intermédio da aplicação do teste $t$ de Student, bicaudal, quanto às médias dos resultados obtidos nos exames gerais referidos no item "Processo Amostral".

A Tabela 1 apresenta média, desvio-padrão, valor mínimo e valor máximo de $\mathrm{Pb}-\mathrm{S}$, ALA-U e ALAD dos trabalhadores dos grupos exposto e não exposto.

* Lefèvre, B.H. Departam ento de Neuropsiquiatria da Faculdade de Medicina da Universidade de São Paulo. São Paulo, comunicação pessoal, 1995. 
Tabela 1- Média, desvio-padrão, valor mínimo e valor máximo de $\mathrm{Pb}-\mathrm{S}, \mathrm{ALA}-\mathrm{U}$ e ALAD dos trabalhadores dos grupos exposto (E) e não exposto (NE).

\begin{tabular}{|c|c|c|c|}
\hline Indicadores & $\begin{array}{c}\text { Média } \\
\text { (desvio-padrão) }\end{array}$ & $\begin{array}{l}\text { Valor } \\
\text { mínimo }\end{array}$ & $\begin{array}{l}\text { Valor } \\
\text { máximo }\end{array}$ \\
\hline $\begin{array}{l}\mathrm{Pb}-\mathrm{S}(\mu \mathrm{g} / \mathrm{dL}) \\
\mathrm{E} \\
\mathrm{NE}\end{array}$ & $\begin{array}{ll}49,44 & (6,83) \\
10,28 & (3,01)\end{array}$ & $\begin{array}{r}35,7 \\
4,5\end{array}$ & $\begin{array}{l}58,8 \\
16,9\end{array}$ \\
\hline $\begin{array}{l}\text { ALA-U }(\mathrm{mg} / \mathrm{L}) \\
\text { E } \\
\text { NE }\end{array}$ & $\begin{array}{ll}3,83 & (2,19) \\
2,72 & (0,93)\end{array}$ & $\begin{array}{l}1,7 \\
0,5\end{array}$ & $\begin{array}{l}9,6 \\
4,1\end{array}$ \\
\hline $\begin{array}{l}\text { ALAD (U/L) } \\
\text { E } \\
\text { NE }\end{array}$ & $\begin{array}{l}25,27(11,39) \\
60,72(10,75)\end{array}$ & $\begin{array}{r}6,6 \\
26,4 \\
\end{array}$ & $\begin{array}{l}39,6 \\
81,6 \\
\end{array}$ \\
\hline
\end{tabular}

Tabela 2 - Média, desvio-padrão, valor mínimo e valor máximo dos resultados das avaliações subteste Vigor do teste Profile of Mood State, Wechsler Memory Scale, subteste Pontilhagem da Bateria Mecânica Léon Walther e subteste Digit Symbol do teste Wechsler Adult Intelligence Scale, obtidos pelos trabalhadores dos grupos exposto (E) e não exposto (NE).

\begin{tabular}{lccc}
\hline Testes & $\begin{array}{c}\text { Média } \\
\text { (desvio-padrão) }\end{array}$ & $\begin{array}{c}\text { Valor } \\
\text { mínimo }\end{array}$ & $\begin{array}{c}\text { Valor } \\
\text { máximo }\end{array}$ \\
\hline Vigor-POMS & $43,65(6,29)$ & 16 & 57 \\
E & $48,45(7,82)$ & 35 & 66 \\
NE & & & \\
WMS & $82,00(15,70)$ & 57 & 105 \\
E & $92,30(12,94)$ & 73 & 122 \\
NE & & & \\
Pont-LW & $38,00(23,21)$ & 10 & 70 \\
E & $56,00(25,21)$ & 10 & 90 \\
NE & & & \\
DS-WAIS & $6,95(1,67)$ & 4 & 10 \\
E & $8,25(2,00)$ & 5 & 12 \\
NE &
\end{tabular}

Vigor-POMS: subteste Vigor do teste Profile of Mood State

WMS: Wechsler Memory Scale

Pont-LW: subteste Pontilhagem do teste Bateria Mecânica Léon Walther

DS-WAIS: subteste Digit Symbol do teste Wechsler Adult Intelligence Scale

Tabela 3 - Comparação entre as médias dos resultados obtidos no subteste Vigor-POMS, no teste Wechsler Memory Scale, no subteste Pontilhagem do teste Bateria Mecânica Léon Walther e no subteste Digit Symbol do teste Wechsler Adult Intelligence Scale entre os trabalhadores dos grupos exposto e controle.

\begin{tabular}{lccc}
\hline & Diferença* $^{*}$ & $\mathrm{t}$ & Valor $\mathrm{p}^{* *}$ \\
\hline Vigor-POMS & $-4,80$ & $-2,1402$ & 0,0194 \\
WMS & $-10,30$ & $-2,2641$ & 0,0147 \\
Pont-LW & $-18,00$ & $-2,3444$ & 0,0122 \\
DC-WAIS & $-1,30$ & $-2,2338$ & 0,0158 \\
\hline
\end{tabular}

Vigor-POMS: resultados obtidos no subteste Vigor do teste Profile of Mood States WMS: resultados obtidos no teste Wechsler Memory Scale

Pont-LW: resultados obtidos no subteste Pontilhagem do Teste Bateria Mecânica Léon Walther

DS-WAIS: resultados obtidos no subteste Digit Symbol do Teste Wechsler Adult Intelligence Scale

* diferença = média entre expostos - média entre não expostos

** valor p calculado com 38 graus de liberdade, assumindo-se variâncias iguais

A Tabela 2 apresenta média, desvio-padrão, valor mínimo e valor máximo dos resultados obtidos pelos trabalhadores dos grupos exposto e não exposto nas avaliações: subteste Vigor do teste Profile of
Mood States, teste Wechsler Memory Scale, subteste Pontilhagem da Bateria Mecânica Léon Walther e subteste Digit Symbol do teste Wechsler Adult Intelligence Scale.

As Figuras 1, 2, 3 e 4 apresentam os boxplots dos resultados obtidos pelos trabalhadores expostos e controles nestes testes e subtestes.

A Tabela 3 resume as estatísticas obtidas por intermédio da comparação das médias dos resultados alcançados nas avaliações neurocomportamentais acima descritas, entre os trabalhadores dos grupos exposto e controle.

O restante dos exames neurocomportamentais realizados pelos grupos exposto e não exposto apresentam médias cujas diferenças não são significativas ao nível $\alpha=0,05$. Tais resultados são encontrados pormenorizadamente descritos em Cordeiro ${ }^{12}$ (1995).

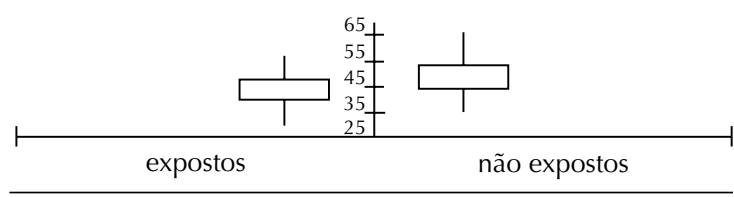

Figura 1 - Boxplot dos resultados do subteste Vigor (Profile of Mood States) entre expostos e não expostos.

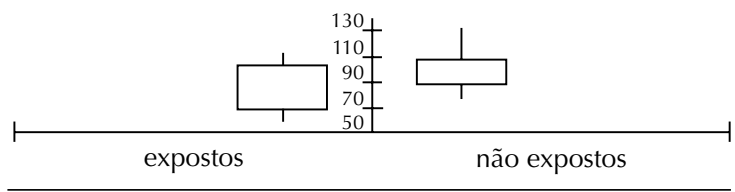

Figura 2 - Boxplot dos resultados do teste Wechsler Memory Scale entre expostos e não expostos.

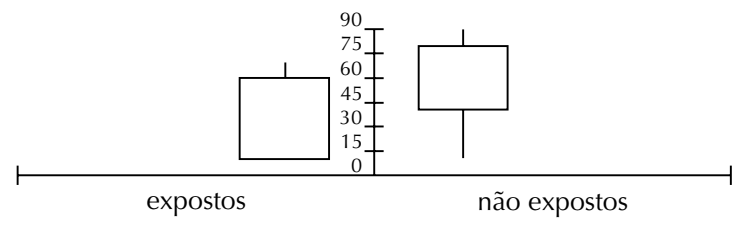

Figura 3 - Boxplot dos resultados do subteste Pontilhagem (Bateria Mecânica Léon Walther) entre expostos e não expostos.

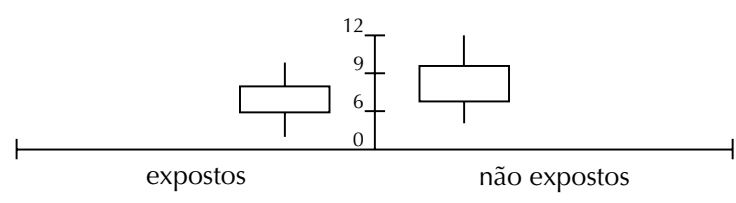

Figura 4 - Boxplot dos resultados do subteste Digit Symbol (Wechsler Adult Inteligence Scale) entre expostos e não expostos. 


\section{DISCUSSÃO}

Conforme a Tabela 3, a maior diferença entre as médias dos resultados das provas neurocomportamentais, referidas na Tabelas 2 , apresenta valor $\mathrm{p}$ igual a 0,0122 ; o que faz rejeitar a hipótese $\mathrm{H}_{0}$ com este nível de significância.

Estes resultados são consonantes com vários trabalhos internacionais, que evidenciaram disfunções da inteligência visual, desempenho visual-motor, formação de conceitos verbais, memória, humor, capacidade de associação verbal de conceitos, compreensão visual e atenção em trabalhadores expostos ao chumbo cujos indicadores $\mathrm{Pb}-\mathrm{S}$ e ALA-U encontravam-se, sistematicamente, abaixo dos valores dos LTB estabelecidos no Brasil 2, 5, 6, 11, 16, 18. Para uma comparação pormenorizada dos resultados aqui apresentados com a literatura internacional especializada, veja-se Cordeiro e Lima-Filho ${ }^{13}$ (1995).

O grupo controle foi selecionado a partir de trabalhadores que exerciam a ocupação de soldadores. Supostamente, alguns desses trabalhadores eventualmente se expunham ao manganês durante alguns procedimentos de solda (solda MIG). Sabese que o manganês é responsável por alterações neurológicas centrais, caracterizadas por um quadro confusional-alucinatório ${ }^{1}$. Não obstante, devido às características de distribuição espacial das seções de trabalho da empresa que forneceu a base populacional do grupo não exposto, a seção dos soldadores foi a melhor escolha possível, basicamente por apresentar número suficientemente grande de trabalhadores, e por colocar-se geograficamente a adequada distância das seções de pintura da montadora, evitando assim a contaminação ocupacional com solventes orgânicos, o que implicaria em problema semelhante, porém de maior monta. Eventualmente, algum efeito desta exposição ao manganês pode ter contribuído para um pior desempenho dos trabalhadores do grupo não-exposto nos testes neurocomportamentais executados (muito embora o serviço médico da referida empresa venha monitorizando periodicamente seus soldadores com relação a possíveis contaminações com

\section{REFERÊNCIAS BIBLIOGRÁFICAS}

1. ADAMS, R.D. \& VICTOR, M. Principles of neurology. 2nd ed. New York, McGraw-Hill Book Company, 1981.

2. ARAKI, S.; YOKOYAMA, K.; AONO, H.; MURATA, K. Psychological performance in relation to central and peripheral nerve conduction in workers exposed to lead, zinc, and copper. Am. J. Ind. Med., 9:535-42, 1986 a. metais pesados, inclusive o manganês, não tendo sido detectado nenhum caso de contaminação até o momento da realização do presente estudo). Tal efeito, se existiu, contribuiu para tornar mais conservador o estudo realizado.

No que diz respeito a avaliações neurocomportamentais, os limites entre o normal e o anormal são imprecisamente conhecidos. Tal imprecisão é ainda ampliada pelo fato de muitas das provas utilizadas não estarem padronizadas para populações brasileiras. Não se pode diagnosticar doença a partir de um desses testes, isoladamente. No entanto, não há como não valorizar o fato de que quatro dos testes aplicados diferenciaram expostos de não expostos. Mesmo porque os testes que resultaram "positivo" - Vigor-POMS, Wechsler Memory Scale, PontilhagemLéon Walther e Digit Symbol-Wechsler Adult Intelligence Scale - avaliam respectivamente humor, memória, coordenação motora e memória, atributos sabidamente sensíveis à ação do chumbo ${ }^{13}$.

Nota-se que - talvez esta seja a maior limitação dos testes neurocomportamentais em toxicologia estes noticiam em geral apenas o resultado final da atividade cognitiva, nada informando sobre a dinâmica de seu processo ${ }^{15}$. Dada a plasticidade do cérebro humano, sua conhecida capacidade de compensação funcional manifesta através de estratégias alternativas de cognição, "inclusive sujeitos que alcançam níveis de execução considerados dentro dos padrões de normalidade podem atingir estes resultados a partir de estratégias compensatórias que evitem a sobrecarga de algum ou alguns sistemas afetados; o que além mascarar o déficit, representa uma elevação do custo psicológico da atividade, sendo fonte de outros transtornos psicológicos e somáticos" $" 15$.

Os resultados e discussão aqui apresentados apontam mais uma vez - como na parte I do presente estudo - contra a validação dos LTB estabelecidos no Brasil para a $\mathrm{Pb}-\mathrm{S}$ e a ALA-U, sugerindo que seus valores, estabelecidos pela Norma Regulamentadora $\mathrm{n}^{\circ} 7^{9}$, atualizada pela Portaria 24/1994 do Ministério do Trabalho ${ }^{8}$, são inadequados para a proteção da saúde dos trabalhadores brasileiros.

3. ARAKI, S.; MURATA, K.; AONO, H. Subclinical cervicospino-bulbar effects of lead: a study of short-latency somatosensory evoked potentials in workers exposed to lead, zinc, and copper. Am. J. Ind. Med., 10: 163-75, 1986 b.

4. BATERIA mecânica Léon Walther: testes de aptidão à mecânica. Rio de Janeiro, Centro de Psicologia Aplicada, s.d. 
5. BAKER, E.L.; FELDMAN, R.G.; WHITE, R.F.; HARLEY, J.P. The role of occupational lead exposure in the genesis of psychiatric and behavioral disturbances. Acta Psychol. Scand., 67 (suppl. 303):38-48, 1983.

6. BAKER, E.L.; FELDMAN, R.G.; WHITE, R.F.; HARLEY, J.P.; NILES, C.A., DINSE, G.E.; BERKEY, C.S. Occupational lead neurotoxicity: a behavioural and electrophysiological evaluation - study design and year one results. $\mathrm{Br} . \mathrm{J}$. Ind. Med., 41: 352-61, 1984.

7. BAKER, E.L.; WHITE, R.F.; POTHIER, L.J.; BERKEY,C.S.; DINSE, G.E.; TRAVERS, P.H.; HARLEY, J.P.; FELDMAN, R.G. Occupational lead neurotoxicity: improvement in behavioural effects after reduction of exposure. Br. J. Ind. Med., 42:507-16, 1985.

8. BRASIL. Leis, etc. Portaria ${ }^{\circ}{ }^{24}$. Diário Oficial da União, Brasília, 30/12/94, 1994. p. 21.

9. BRASIL. Ministério do Trabalho. Norma Regulamentadora $\mathrm{n}^{\circ}$ 7. In: Pimenta, A.L. \& Capistrano F ${ }^{\circ}$, D., org. Saúde do trabalhador. São Paulo, Hucitec, 1988. p. 144-52.

10. CAMBRAIA, S.V. Teste atenção concentrada. s.l., Vetor Editora Psico-pedagógica, 1967.

11. CAMPARA, P.; D'ANDREA, F.; MICCIOLO, R.; SAVONITTO, C.; TANSELLA, M.; TANSELLA, Z. Psychological performance of workers with blood-lead concentration below the current threshold limit value. Int Arch. Occup. Environ. Health., 53:233-46, 1984.
12. CORDEIRO, R. Quando começa o saturnismo? Campinas, 1995. [Tese de Doutorado - Faculdade de Ciências Médicas da UNICAMP].

13. CORDEIRO, R. \& LIMA FILHO, E.C. A inadequação dos valores dos limites de tolerância biológica para a prevenção da intoxicação profissional pelo chumbo no Brasil. Cad. Saúde Pública, 11:177-86, 1995.

14. CORDEIRO, R; LIMA FILHO, E.C.; SALGADO, P.E.T. Distúrbios neurológicos em trabalhadores com baixos níveis de chumbo no sangue. I- Neuropatia periférica. Rev. Saúde Pública, 30:248-55, 1996.

15. COSENZA, G.W. Efectos neurotóxicos del plomo: indicadores subclínicos. La Habana, 1989. [Maestria en Salud Ocupacional. Ministério de Salud Pública].

16. MANTERE, P.; HÄNINEN, H.; HERNBERG, S.; LUUKKONEN, R. A prospective follow-up study on psychological effects in workers exposed to low levels of lead. Scand. J. Work Environ. Health, 10:43-50, 1984.

17. McNAIR, D.M.; LORR, M.; DROPPELMAN, F. EITS manual for profile of mood states. San Diego, Educational and Industrial Testing Service, 1971.

18. STOLLERY, B.T. Cognitive function in lead workers. $\mathrm{Br}$. $J$. Ind. Med., 46:698-707, 1989.

19. WORLD HEALTH ORGANIZATION. Operational guide for the WHO neurobehavioral core test battery. Geneva, Office of Occupational Health, 1986. 